\title{
BMJ Randomised controlled trial comparing OPOn hypnotherapy versus gabapentin for the treatment of hot flashes in breast cancer survivors: a pilot study
}

\author{
Shannon MacLaughlan David, ${ }^{1}$ Sandra Salzillo, ${ }^{2}$ Patrick Bowe, ${ }^{3}$ Sandra Scuncio, ${ }^{2}$ \\ Bridget Malit, ${ }^{4}$ Christina Raker, ${ }^{2}$ Jennifer S Gass, ${ }^{2}$ C O Granai, ${ }^{2}$ Don S Dizon ${ }^{5}$
}

To cite: MacLaughlan David S, Salzillo S, Bowe P, et al. Randomised controlled trial comparing hypnotherapy versus gabapentin for the treatment of hot flashes in breast cancer survivors: a pilot study. BMJ Open 2013;3:e003138. doi:10.1136/bmjopen-2013003138

- Prepublication history for this paper is available online. To view these files please visit the journal online (http://dx.doi.org/10.1136/ bmjopen-2013-003138).

Received 28 April 2013 Revised 15 July 2013 Accepted 2 August 2013

For numbered affiliations see end of article.

\section{Correspondence to}

Dr Shannon MacLaughlan David;

smaclaug@stanford.edu

\section{ABSTRACT}

Objectives: To compare the efficacy of hypnotherapy versus gabapentin for the treatment of hot flashes in breast cancer survivors, and to evaluate the feasibility of conducting a clinical trial comparing a drug with a complementary or alternative method (CAM).

Design: Prospective randomised trial.

Setting: Breast health centre of a tertiary care centre.

Participants: 15 women with a personal history of breast cancer or an increased risk of breast cancer who reported at least one daily hot flash.

Interventions: Gabapentin $900 \mathrm{mg}$ daily in three divided doses (control) compared with standardised hypnotherapy. Participation lasted 8 weeks.

Outcome measures: The primary endpoints were the number of daily hot flashes and hot flash severity score (HFSS). The secondary endpoint was the Hot Flash Related Daily Interference Scale (HFRDIS).

Results: 27 women were randomised and $15(56 \%)$ were considered evaluable for the primary endpoint $(n=8$ gabapentin, $n=7$ hypnotherapy). The median number of daily hot flashes at enrolment was 4.5 in the gabapentin arm and 5 in the hypnotherapy arm. HFSS scores were 7.5 in the gabapentin arm and 10 in the hypnotherapy arm. After 8 weeks, the median number of daily hot flashes was reduced by $33.3 \%$ in the gabapentin arm and by $80 \%$ in the hypnotherapy arm. The median HFSS was reduced by $33.3 \%$ in the gabapentin arm and by $85 \%$ in the hypnotherapy arm. HFRDIS scores improved by $51.6 \%$ in the gabapentin group and by $55.2 \%$ in the hypnotherapy group. There were no statistically significant differences between groups.

Conclusions: Hypnotherapy and gabapentin demonstrate efficacy in improving hot flashes. A definitive trial evaluating traditional interventions against CAM methods is feasible, but not without challenges. Further studies aimed at defining evidencebased recommendations for CAM are necessary. Trial registration: clinicaltrials.gov (NCT00711529).

\section{INTRODUCTION}

Breast cancer is the most common cancer among women, with more than 230000 anticipated new cases in 2013. ${ }^{1}$ Owing to

\section{ARTICLE SUMMARY}

Strengths and limitations of this study

- This is the first investigation comparing hypnotherapy to a standard-of-care treatment for hot flashes in breast cancer survivors. Our results add to the accumulating body of evidence supporting the use of CAM for symptom management in cancer survivors.

- This pilot study could not demonstrate statistical differences between the two treatment groups because of the small numbers. Nonetheless, the findings are clinically relevant. Our experience elucidated potential barriers in the design and implementation of phase III trials evaluating CAM against current standards of care.

improvements in screening, diagnosis and therapy for breast cancer, the survival rate continues to improve and there are now more than 2.5 million breast cancer survivors in the USA. ${ }^{2}$

Among breast cancer survivors, hot flashes are a frequent and bothersome symptom. Up to $90 \%$ of young breast cancer survivors experience premature menopause because of adjuvant treatment strategies such as chemotherapy, antioestrogenic agents and ovarian ablation techniques. In addition to therapy-induced hot flashes, these vasomotor symptoms are common among breast cancer survivors undergoing natural menopause. ${ }^{3-5}$

Hot flashes have been shown to have a significant impact on women's mood and sleep, and among breast cancer survivors, they are associated with deficiencies in the quality of life and survivorship. ${ }^{6-8}$

As hot flashes are caused by oestrogen deprivation, the most effective treatment for these vasomotor symptoms is oestrogen replacement therapy. However, despite a lack of data demonstrating an increased risk of 
developing primary or recurrent breast cancer from oestrogen replacement alone, its use in breast cancer survivors or women at risk for developing breast cancer is largely considered a contraindication. ${ }^{9}$ Even among women whose medical oncologists feel comfortable prescribing oestrogen replacement, less than a third are willing to try it. ${ }^{10}$

Non-hormonal drug therapies for the treatment of hot flashes have demonstrated moderate efficacy but are associated with side effects. The most commonly prescribed agents are antidepressants, which have been shown to reduce self-reported hot flashes in breast cancer survivors by up to $61 \% \cdot{ }^{11-18}$ However, antidepressants may interfere with the metabolism of tamoxifen, a common treatment for oestrogen receptor-positive breast cancers, by inhibiting the CYP2D6 enzyme. ${ }^{19}$ Venlafaxine is the most commonly prescribed antidepressant for hot flashes and has only minimal inhibition of cyp2D6 without known clinical significance. However, gabapentin, a $\gamma$-amino-butyric acid analogue commonly used for the treatment of seizure disorders and neuropathic pain, has no effect on cyp2D6 metabolism. ${ }^{20}$

The efficacy of gabapentin to manage hot flashes was demonstrated in women undergoing natural menopause, showing comparable results as oestrogen replacement. ${ }^{21}$ In a randomised trial against a placebo, hot flashes were decreased by $51 \%$ in those treated with gabapentin, compared with $26 \%$ in those treated with placebo $(\mathrm{p}<0.001)$. While dizziness, unsteadiness and drowsiness were reported in the first week among women taking gabapentin, symptoms resolved within 4 weeks. ${ }^{22}$

Gabapentin was further validated as a treatment for hot flashes in breast cancer survivors, including women already taking an antidepressant. ${ }^{23-25}$ In a definitive study of 420 women who were randomised to receive gabapentin $300 \mathrm{mg} /$ day, gabapentin $900 \mathrm{mg} /$ day or placebo, the $900 \mathrm{mg} /$ day dose of gabapentin was the most effective, decreasing the frequency of hot flashes by $41 \%$ at 4 weeks compared with $17 \%$ reduction in the placebo group. However, in this same treatment group, there was a $12 \%$ withdrawal rate at 4 weeks, and $17 \%$ at 8 weeks, owing to side effects and subjective inefficacy. ${ }^{24}$

The need for non-pharmaceutical treatment options for hot flashes in cancer survivors is evident by the high dropout rates seen in these drug studies. Limited options for safe and effective non-hormonal drugs and increasing interest among women in complementary and alternative medicine (CAM) have led to the need for investigation of non-pharmacological modalities.

Hypnosis is a mind-body therapy involving the induction of a state of deep relaxation that allows the patient to suspend critical faculties and allow suggestibility. In a pilot study evaluating hypnotherapy for the treatment of hot flashes in breast cancer survivors, 16 women experienced a 59\% reduction in hot flash frequency. ${ }^{26}$ In a randomised trial comparing hypnotherapy to no treatment, 60 female breast cancer survivors were randomised to receive either hypnosis or no treatment. The women who underwent hypnotherapy reported a reduction in hot flash severity scores (HFSS) of $68 \%$, and also reported improvement in quality of life variables such as insomnia. ${ }^{27}$ Women receiving hypnotherapy did not report side effects in either trial.

Hypnotherapy has been further validated as a treatment of hot flashes in a recent clinical trial that randomised 187 postmenopausal women to undergo clinical hypnosis or structured-attention control. ${ }^{28}$ Women in the treatment group experienced a $74 \%$ reduction in hot flash frequency compared with a $17 \%$ reduction in the control group. These authors devised a structured-attention exposure to therapists to serve as a placebo-like control. However, clinical trials evaluating CAM modalities are often limited by inadequate control groups, and hypnotherapy has never been compared directly to a standard therapy in the treatment of hot flashes.

To address the needs of the growing population of breast cancer survivors, we designed this study to assess the feasibility of a prospective randomised controlled trial evaluating the efficacy of hypnotherapy versus gabapentin for the treatment of hot flashes in women with a personal history or increased risk of developing breast cancer.

\section{PATIENTS AND METHODS}

Women were enrolled and monitored in the breast health centre of a tertiary care centre (Women and Infants Hospital, Providence, Rhode Island, USA).

Eligible patients were at least 18 years of age and had a history of breast cancer without evidence of current disease. To improve accrual, eligibility was expanded to include women who had an increased risk of developing breast cancer in their lifetime. 'Increased risk' was defined as age greater than 60, BRCA mutation or strong family history, history of preinvasive breast lesion or a lifetime Gail model score of $1.6 \%$ or greater. Women taking antioestrogen therapy such as tamoxifen or aromatase inhibitors were included. Eligible women reported at least one daily hot flash. Women with persistent daily hot flashes undergoing other treatments, such as medications or counselling, were included as long as they were not taking gabapentin or undergoing hypnotherapy. All participants were asked not to initiate new therapy for hot flashes during the study period.

Women were excluded who were undergoing chemotherapy or radiation for their breast cancer, or who had serious psychiatric or medical illness that may interfere with participation in the protocol. All patients provided written informed consent.

Participants were randomised to receive gabapentin in the control arm or standardised hypnotherapy in the experimental arm based on a block randomisation scheme generated at http://www.randomization.com. Participation lasted 8 weeks in both arms.

Women assigned to the control arm were prescribed gabapentin $900 \mathrm{mg}$ daily by the principal investigator, in three divided doses. ${ }^{24}$ Patients randomised to the 
experimental arm were scheduled for three $1 \mathrm{~h}$ hypnotic inductions by a single certified hypnotherapist, each 1 week apart. Standardised outlines were used for each induction. The first session consisted of an induction directed towards complete relaxation and suggesting that the woman can control her symptoms with her unconscious mind. The second and third sessions also began with a standardised induction, followed by the establishment of an 'anchor', or physical reference point (forefinger to thumb), used to invoke images of coolness, which were individualised according to patient preference. Patients were also instructed by the same hypnotherapist in self-hypnosis and guided imagery techniques to be used at home with the assistance of standardised audio CDs.

Women were asked to keep a daily hot flash diary for the 8 weeks of participation. ${ }^{29}$ Quality of life data were collected with the Hot Flash Related Daily Interference Scale (HFRDIS) ${ }^{30}$ conducted on the day of enrolment, and at 4-week and 8-week follow-up visits.

The primary endpoints for this study were daily frequency of hot flashes and HFSS, both of which were derived from participants' diaries. HFSS were calculated by assigning one point to each mild hot flash, two points for each moderate hot flash, three points for each severe hot flash and four points for each very severe hot flash. The secondary endpoint was HFRDIS scores. A modified intention-to-treat analysis was performed, including all data provided by the participants randomised. Fisher's exact test was used to compare categorical variables and the Wilcoxon rank-sum test/Mann-Whitney U test was used to compare continuous variables using the SAS V.9.2 statistical package (SAS Institute, Cary, North Carolina, USA).

A power analysis was conducted to determine the sample size needed for a definitive study. Based on an $80 \%$ power to detect a doubling of effect in the experimental arm compared with control (with $\alpha=0.05$ ), a sample size of 64 women in each arm would be necessary. In this pilot feasibility study, we set a goal of recruiting 60 women into participation. We concluded enrolment after a predetermined 2-year time period.

\section{RESULTS}

Between September 2008 and September 2010, 73 women were screened for this study. Fourteen women were deemed ineligible and 32 decided not to participate. A total of 27 women were randomised to receive treatment, 14 to gabapentin and 13 to hypnotherapy (figure 1). Two women randomised to receive hypnotherapy were disqualified: one did not speak English and could therefore not comply with the hypnotherapy protocol, and the other had active metastatic disease. A total of five patients dropped out prior to initiating treatment. Three did not want to take gabapentin, and two could not fit hypnotherapy sessions into their work schedules.

Eleven patients initiated gabapentin therapy, and eight completed the study. The three women who discontinued the drug cited side effects and inefficacy as their reasons. These three women declined further participation in the study and did not submit their hot flash diaries.

Nine patients initiated hypnotherapy. One patient underwent two of three inductions; otherwise, all nine completed the study protocol. All nine were included in the analysis; however, two women in the hypnotherapy arm were not evaluable for the primary endpoint because of lost hot flash diaries.

Demographic data of the two groups are presented in table 1 . There were no significant differences between the groups.

Hot flash frequencies and HFSS were derived from the submitted diaries. Data were available for eight women in the gabapentin arm and seven women in the hypnotherapy arm. The median number of daily hot flashes at enrolment was 4.5 in the gabapentin arm and 5 in the hypnotherapy arm (table 2A). After 8 weeks, the median number of daily hot flashes was reduced by $33.3 \%$ in the gabapentin arm and by $80 \%$ in the hypnotherapy arm (figure 2). The median HFSS at baseline was 7.5 in the gabapentin arm $(n=8)$ and 10 in the hypnotherapy arm $(\mathrm{n}=7$; table $2 \mathrm{~B})$. The median HFSS was reduced by $33.3 \%$ in the gabapentin arm and by $85 \%$ in the hypnotherapy arm (figure 3 ). These do not represent statistically significant differences between treatment arms.

Baseline HFRDIS data were available for all women who initiated therapy, and scores for the two treatment groups were similar (table 2C). There was no difference in treatment effect between the two groups. Overall, the HFRDIS scores improved by $51.6 \%$ in the gabapentin group and by $55.2 \%$ in the hypnotherapy group.

Sensitivity analyses were performed for all endpoints, imputing values for non-responders that assumed greater and lesser improvement. Observed differences in treatment effects still did not reach statistical significance.

\section{DISCUSSION}

The findings of our study suggest the efficacy of hypnotherapy and gabapentin with a noteworthy trend towards a benefit for hypnosis. While not statistically significant, we believe that these results are clinically important and warrant further investigation.

None of the women in the hypnotherapy arm reported adverse effects of their treatment, and all women reported that they felt the hypnosis worked. However, two women were never able to undergo hypnotherapy because of scheduling conflicts, and a third woman only had two of three planned sessions for the same reason. Conversely, three women in the gabapentin arm dropped out after randomisation and before starting the drug, and three dropped out due to side effects (worsening vertigo and fatigue) and inefficacy. This is consistent with the anticipated dropout rate 


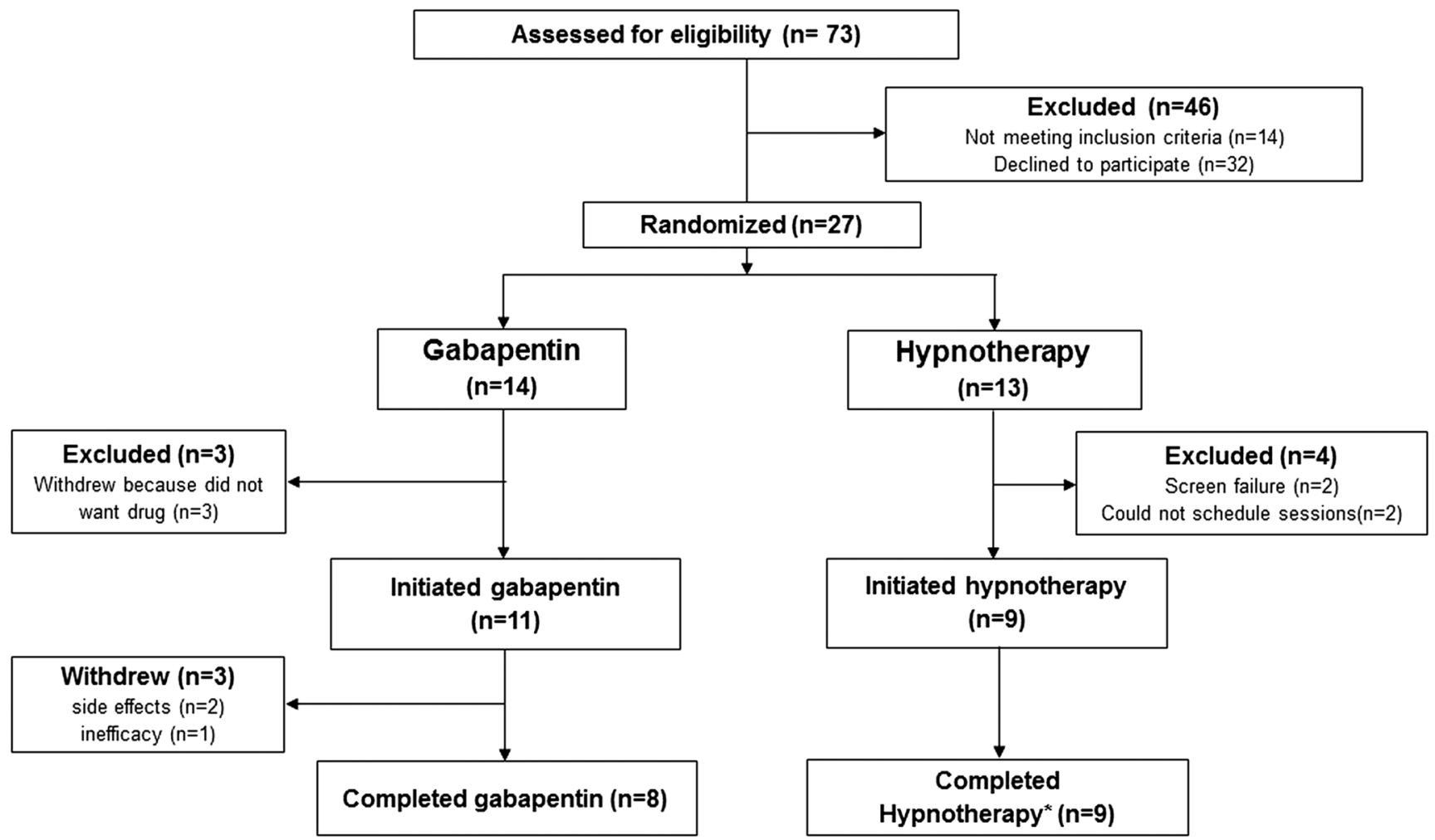

Figure 1 Consort flow diagram for screening and enrolment. *Two patients lost their hot flash diaries and therefore were not evaluable for the primary endpoint.

based on clinical trials evaluating medications for the treatment of hot flashes. ${ }^{21} 2224$

In women undergoing hypnotherapy, we report an $80 \%$ improvement in hot flash frequency and an $85 \%$ improvement in HFSS. This treatment effect is similar to that in previous studies evaluating hypnotherapy, and apparently stronger than what has been reported previously for gabapentin. ${ }^{21-28}$ However, caution is necessary in comparing results across studies. For example, Elkins's most recent clinical trial among postmenopausal women required a minimum of seven daily hot flashes. That study was able to demonstrate a $74 \%$ reduction in hot flashes compared with $17 \%$ in the control group. In contrast, in the definitive study demonstrating the efficacy of gabapentin, women were included who had at least two daily hot flashes and demonstrated a $44 \%$ reduction in hot flash frequency over 8 weeks (compared with $15 \%$ in control)..$^{24}$ In a population with more frequent and more severe hot flashes, a treatment effect is easier to demonstrate, and this must be taken into account when examining eligibility criteria and comparing the results of one trial with that of another. A direct comparison in a definitive trial is necessary to determine superiority because of the significant

\begin{tabular}{|c|c|c|c|}
\hline & $\begin{array}{l}\text { Gabapentin } \\
n=14\end{array}$ & $\begin{array}{l}\text { Hypnotherapy } \\
\mathrm{n}=11\end{array}$ & p Value ${ }^{\star}$ \\
\hline Median age & $52(45,63)$ & $56(42,62)$ & - \\
\hline \multicolumn{4}{|l|}{ Race } \\
\hline Caucasian & $14(100 \%)$ & $10(90.9 \%)$ & \multirow[t]{2}{*}{0.4} \\
\hline African-American & 0 & $1(9.1 \%)$ & \\
\hline History of cancer & $11(78.6 \%)$ & $5(45.5 \%)$ & 0.1 \\
\hline Tamoxifen & $6(42.9 \%)$ & $3(27.3 \%)$ & 0.7 \\
\hline Antidepressant use & $3(21.4 \%)$ & $2(18.2 \%)$ & 1.0 \\
\hline \multicolumn{4}{|c|}{ Baseline daily hot flashes } \\
\hline $1-5$ & $3(21.4 \%)$ & $1(9.1 \%)$ & \multirow[t]{3}{*}{0.8} \\
\hline $5-10$ & $8(57.1 \%)$ & $8(72.7 \%)$ & \\
\hline$>10$ & $3(21.4 \%)$ & $2(18.2 \%)$ & \\
\hline
\end{tabular}


Table 2 Comparison of primary and secondary endpoints

\begin{tabular}{|c|c|c|c|}
\hline \multicolumn{4}{|l|}{ A. Hot flash frequencies } \\
\hline & \multicolumn{2}{|c|}{ Number of daily hot flashes } & \multirow[b]{2}{*}{ p Value* } \\
\hline & $\begin{array}{l}\text { Hypnotherapy }(\mathrm{n}=7) \\
\text { Median (range); } \mathrm{n}\end{array}$ & $\begin{array}{l}\text { Gabapentin }(\mathrm{n}=8) \\
\text { Median (range); } \mathrm{n}\end{array}$ & \\
\hline Baseline & $5.0(2,11) ; 7$ & $4.5(2,9) ; 8$ & 1.0 \\
\hline Week 4 & $4.0(1,7) ; 6$ & $4.0(2,9) ; 7$ & 0.7 \\
\hline Week 8 & $1.0(0,7) ; 6$ & $3.0(3,5.5) ; 5$ & 0.3 \\
\hline Percentage of decrease $†$ & $80 \%$ & $33.3 \%$ & \\
\hline \multicolumn{4}{|c|}{ B. Hot flash severity scores (HFSSs) } \\
\hline & \multicolumn{2}{|l|}{ HFSS } & \multirow[b]{3}{*}{ p Value* } \\
\hline & Hypnotherapy (n=7) & Gabapentin $(n=8)$ & \\
\hline & Median (range); $\mathbf{n}$ & Median (range); $\mathbf{n}$ & \\
\hline Baseline & $10.0(2.0,14.0) ; 7$ & $7.5(2.0,19.0) ; 8$ & 0.7 \\
\hline Week 4 & $6.5(1.0,11.0) ; 6$ & $4.0(2.0,17.5) ; 7$ & 0.6 \\
\hline Week 8 & $1.5(0,12.0) ; 6$ & $5.0(3.0,11.5) ; 5$ & 0.3 \\
\hline Percentage of decrease & $85 \%$ & $33.3 \%$ & \\
\hline \multicolumn{4}{|c|}{ C. Hot Flash Related Daily Interference Scale (HFRDIS) scores } \\
\hline & \multicolumn{2}{|l|}{ HFRDIS } & \multirow[b]{3}{*}{ p Value* } \\
\hline & Hypnotherapy $(n=11)$ & Gabapentin $(n=14)$ & \\
\hline & Median (range); $\mathbf{n}$ & Median (range); $\mathbf{n}$ & \\
\hline Baseline & $58.0(16,64) ; 11$ & $45.5(11,82) ; 14$ & 0.8 \\
\hline Week 4 & $25.5(1,57) ; 6$ & $12.5(0,57) ; 8$ & 0.8 \\
\hline Week 8 & $26.0(0,68) ; 9$ & $22.0(7,41) ; 7$ & 0.9 \\
\hline Percentage of decrease & $55.2 \%$ & $51.6 \%$ & \\
\hline
\end{tabular}

heterogeneity of hot flash characteristics in the different populations studied.

Women are frequent users of CAM therapies and have reported using such modalities for menopausal symptoms. A survey of 886 women aged 45-65 years reported that $76.1 \%$ were using some kind of CAM therapy and $22.1 \%$ were using it specifically for menopausal symptoms. ${ }^{31}$ More than half of the breast cancer survivors reported using CAM in the Nurses' Health Study. ${ }^{32}$ In the light of the increasing interest in CAM therapies

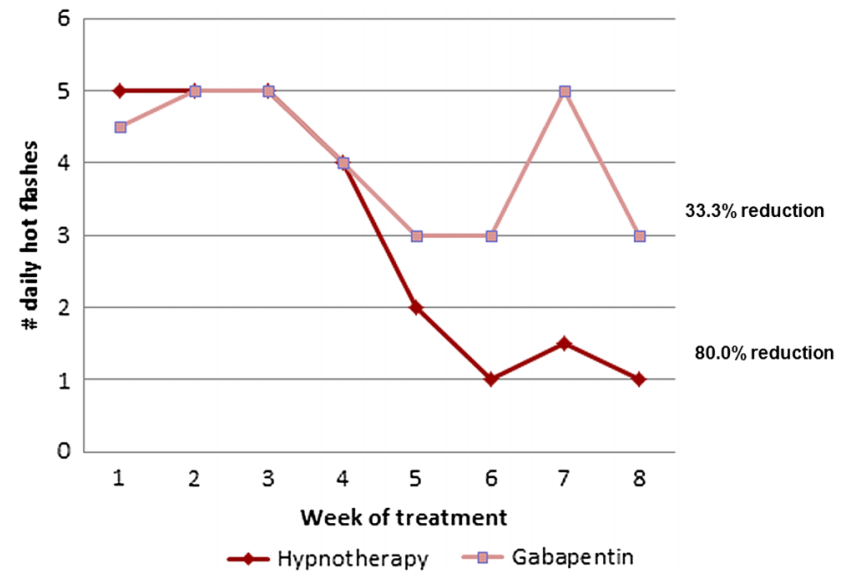

Figure 2 Median number of daily hot flashes. among women and cancer survivors and the National Cancer Institute's Initiative to expand the goal of clinical trials to address symptom management in cancer care, a need has arisen for clinical trials evaluating CAM modalities for the treatment of cancer and treatment-related symptoms. In the study presented here, we explored the feasibility of comparing the efficacy of a CAM modality in a prospective randomised clinical trial with a standard pharmacological therapy as a control for the treatment of hot flashes.

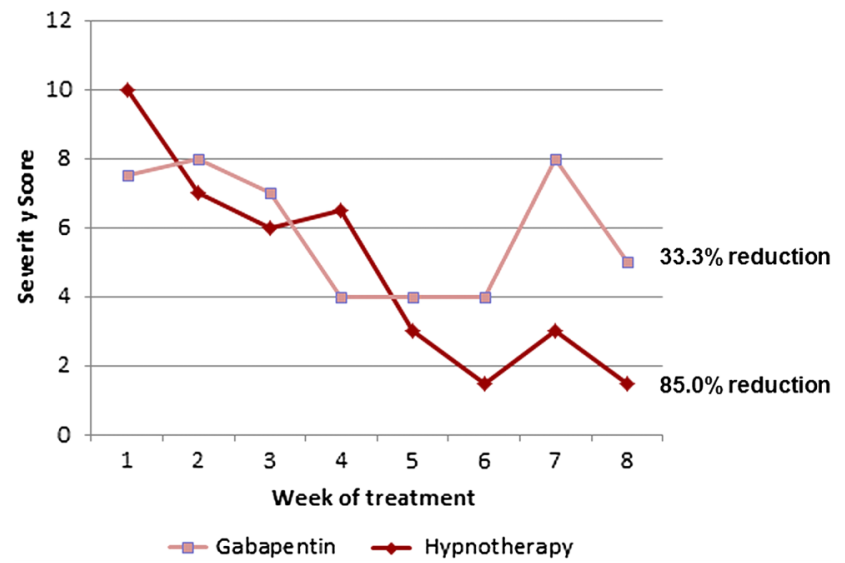

Figure 3 Median hot flash severity scores. 
Our experience has confirmed the feasibility of such a trial, but several points are worthy of discussion regarding the study design.

A significant deficit of trials investigating CAM therapies is the lack of an adequate control group. ${ }^{33}$ For example, there is no 'placebo' equivalent to therapies such as hypnotherapy, and patients and providers cannot be blinded to whether patients are undergoing hypnosis. Elkins et al accounted for this in their study evaluating breast cancer survivors by controlling their randomised trial with a group of women who were placed on a waiting list for hypnotherapy. The women in the control arm experienced no significant change in their hot flashes, consistent with a 'no treatment' intervention. ${ }^{27}$ However, in the case of hot flashes, there is a well-established placebo effect of $20-30 \%$ observed in clinical trials evaluating medications, suggesting that 'no treatment' arms may not be adequate controls either. ${ }^{29}$ In their more recent trial evaluating hypnotherapy for hot flashes in postmenopausal women, Elkins $e t a l^{28}$ used a structured-attention control designed to mimic the hypnotherapy intervention without therapeutic intent. This allowed for a singleblinded model, and the 'placebo' effect was $17 \%$, similar to that seen in the control arm of the drug trials.

It is difficult to control for a provider bias with a single-blinded study, and comparing treatment effect results between trials is an unreliable method as discussed. Therefore, we advocate comparing hypnosis directly with an evidence-based standard therapy in a definitive study. However, we encountered significant obstacles to accrual with such a study design.

In our 2 years of recruitment, we were able to formally screen only 73 women. We hypothesise the low rate of referral to be secondary to a deficit in knowledge regarding CAM among referring physicians. In a separate study, we queried healthcare providers who care for breast cancer survivors regularly. Every provider reported a willingness to refer patients to a clinical trial involving CAM, but only $25 \%$ felt qualified to counsel a patient regarding CAM modalities (Malit $e t$ al, NEAGO 2010).

In addition, among the women screened, the majority was deemed eligible $(n=59)$, but less than half were willing to undergo randomisation because of a strong preference to avoid taking a medication. Among the 14 women randomised to the gabapentin arm, only $8 \mathrm{com}$ pleted treatment. Our experience suggests an inherent bias among women regarding their treatment options. This was confirmed in a survey of breast health patients in which $67 \%$ reported their willingness to participate in a clinical trial evaluating a CAM modality, and only $48 \%$ would agree to a trial in which a medication was the control (Malit et al, NEAGO 2010). The majority of these women reported that their cancer-related symptoms were not severe enough to undergo any treatment, emphasising the need to distinguish daily hot flashes from bothersome hot flashes.

Limited accrual necessitates broad eligibility criteria in study design. We included any woman with a personal history of breast cancer or an increased risk of developing breast cancer over her lifetime. Though the objective of our research was to address cancer-related symptoms, the issue of therapy-induced hot flashes is also relevant for women taking the prophylactic tamoxifen. Bothersome hot flashes, whether treatment related or not, are a common problem among all women, and a larger clinical trial would allow for detection of potential differences in effect among breast cancer survivors and other women. In addition, it is common practice for investigators to include all women with a 'real or perceived risk' of breast cancer related to oestrogen replacement in clinical trials investigating hot flash therapies. ${ }^{16} 222729$

Also in an effort to maximise accrual, we chose to include women who reported at least one daily hot flash. This is a lower threshold of hot flash frequency required for enrolment than other studies, resulting in lower baseline daily hot flash frequencies of 4.5 in the gabapentin arm and 5 in the hypnotherapy arm. ${ }^{11}{ }^{22-27}$ The lower baseline frequency may explain why the treatment effect in the gabapentin arm was only $33.3 \%$, lower than previous studies and more consistent with that in the placebo arm in most clinical trials evaluating hot flashes. ${ }^{23-25} 29$

Our study also included women who were already on therapies for their hot flashes or medications that could affect their hot flashes, such as antidepressants. These treatments could be considered as confounding variables in determining the treatment effect of our study interventions. However, these women still had to meet the eligibility criterion of one daily hot flash and were asked to avoid making changes in their therapies during the study period. This allowed for evaluation of our treatment modalities among women who were suffering persistent hot flashes despite other therapies, an approach supported by previous studies. ${ }^{25}$

Sufficient data exist for the use of either gabapentin or hypnotherapy for the treatment of hot flashes in women who cannot or will not take oestrogen replacement for the treatment of hot flashes. However, a larger definitive trial is justified despite the challenges for a number of reasons. Further research evaluating CAM compared with standard therapies will provide evidence needed by many providers to make referrals, a safer approach than patients referring themselves for hypnosis. Further education in CAM modalities among physicians would improve accrual and recruitment into clinical trials. A larger study would also provide the opportunity to investigate whether there are predictive variables for the efficacy of each modality or specific effects on quality of life variables, which would allow for long-term follow-up evaluations, and which are lacking in the current literature on hot flash therapies.

The use of hormone replacement and the associated risk of recurrent or new breast cancer is controversial. However, a growing number of women are either unwilling or unable to take oestrogen replacement therapy for treatment of their vasomotor symptoms, and many are not interested in taking medications. Hypnotherapy is a CAM 
modality with no known adverse effects; it is a promising, safe and effective alternative to pharmacological therapies for hot flashes. Further studies evaluating hypnotherapy compared with standard therapies are needed.

\section{Author affiliations}

${ }^{1}$ Department of Obstetrics and Gynecology, Stanford Women's Cancer Center, Stanford University, Stanford, California, USA

${ }^{2}$ Program in Women's Oncology, Women \& Infants Hospital, Alpert Medical School at Brown University, Providence, Rhode Island, USA

${ }^{3}$ Providence Hypnosis Center, Providence, Rhode Island, USA

${ }^{4}$ Department of Pediatrics, Weill Cornell Medical College of Cornell University, New York, New York, USA

${ }^{5}$ Department of Internal Medicine, Massachusetts General Hospital, Harvard University, Boston, Massachusetts, USA

Contributors SMD, DSD, JSG and COG were responsible for the conception and implementation of the study. SMD and DSD designed the study and were responsible for interpretation of results. SMD, DSD and PB wrote the protocol. SMD, SSa, SSc and BM recruited, consented and enrolled patients. PB performed the hypnotherapy. Data collection was performed by SMD and SSa. CR and SMD were responsible for data analysis and generation of figures and tables. SMD wrote the initial draft of the manuscript and circulated it for critical review. Major revisions were provided by DSD. All authors reviewed and approved the final version of the manuscript.

Funding This research received no specific grant from any funding agency in the public, commercial or not-for-profit sectors.

Competing interests PB is the director and proprietor of the Providence Hypnosis Center. He was compensated (at a reduced rate) for the hypnotherapy sessions administered.

Ethics approval This randomised controlled trial was approved by the Institutional Review Board, Women and Infants Hospital and was registered with clinicaltrials.gov (NCT00711529).

Provenance and peer review Not commissioned; externally peer reviewed.

Data sharing statement No additional data are available.

Open Access This is an Open Access article distributed in accordance with the Creative Commons Attribution Non Commercial (CC BY-NC 3.0) license, which permits others to distribute, remix, adapt, build upon this work noncommercially, and license their derivative works on different terms, provided the original work is properly cited and the use is non-commercial. See: http:// creativecommons.org/licenses/by-nc/3.0/

\section{REFERENCES}

1. American Cancer Society. Cancer facts \& figures. Atlanta: American Cancer Society, 2013.

2. Frieden TR, Jaffe HW, Stephens JW, et al. Cancer survivors-United States, 2007. MMWR Morb Mortal Wkly Rep 2011;60:269-72.

3. Goodwin PJ, Ennis M, Pritchard KI, et al. Risk of menopause during the first year after breast cancer diagnosis. J Clin Oncol 1999;17:2365-70.

4. Bachmann GA. Vasomotor flushes in menopausal women. Am J Obstet Gynecol 1999;180:S312-16.

5. Carpenter JS, Andrykowski MA, Cordova M, et al. Hot flashes in postmenopausal women treated for breast carcinoma: prevalence, severity, correlates, management, and relation to quality of life. Cancer 1998;82:1682-91.

6. Avis NE, Crawford S, Manuel J. Quality of life among younger women with breast cancer. J Clin Oncol 2005:23:3322-30.

7. Knobf MT. The influence of endocrine effects of adjuvant therapy on quality of live outcomes in younger breast cancer survivors. Oncologist 2006;11:96-110.

8. Ganz PA, Greendale GA, Petersen L, et al. Breast cancer in younger women: reproductive and late health effects of treatment. J Clin Oncol 2003;21:4184-93.
9. Pritchard $\mathrm{KI}$. The role of hormone replacement therapy in women with a previous diagnosis of breast cancer and a review of possible alternatives. Ann Oncol 2001:12:301-10.

10. Couzi RJ, Helzlsouer KJ, Fetting JH. Prevalence of menopausal symptoms among women with a history of breast cancer and attitudes toward estrogen replacement therapy. J Clin Oncol 1995;13:2737-44.

11. Loprinzi CL, Pisansky TM, Fonseca R, et al. Pilot evaluation of venlafaxine hydrochloride for the therapy of hot flashes in cancer survivors. J Clin Oncol 1998;16:2377-81.

12. Loprinzi CL, Kugler JW, Sloan JA, et al. Venlafaxine in management of hot flashes in survivors of breast cancer: a randomised controlled trial. Lancet 2000;356:2059-63.

13. Barton D, LaVasseur B, Loprinzi C, et al. Venlafaxine for the control of hot flashes: results of a longitudinal continuation study. Oncol Nurs Forum 2002;29:33-40.

14. Carpenter JS, Storniolo AM, Johns S, et al. Randomized, double-blind, placebo-controlled crossover trials of venlafaxine for hot flashes after breast cancer. Oncologist 2007;12:124-35.

15. Kimmick GG, Lovato J, McQuellon R, et al. Randomized, double-blind, placebo-controlled, crossover study of sertraline for the treatment of hot flashes in women with early stage breast cancer taking tamoxifen. Breast J 2006;12:114-22.

16. Stearns V, Slack R, Greep N, et al. Paroxetine is an effective treatment for hot flashes: results from a prospective randomized clinical trial. J Clin Oncol 2005;23:6919-30.

17. Loprinzi CL, Sloan JA, Perez EA, et al. Phase III evaluation of fluoxetine for treatment of hot flashes. J Clin Oncol 2002;20:1578-83.

18. Barton DL, Loprinzi CL, Novotny P, et al. Pilot evaluation of citalopram for the relief of hot flashes. $J$ Support Oncol 2003;1:47-51.

19. Jin Y, Desta Z, Stearns V, et al. Cyp2D6 genotype, antidepressant use, and tamoxifen metabolism during adjuvant breast cancer treatment. J Natl Cancer Inst 2005;97:30-9.

20. Benedetti MS. Enzyme induction and inhibition by new antiepileptic drugs: a review of human studies. Fundam Clin Pharmacol 2000;14:301-19.

21. Reddy SY, Warner H, Guttuso, et al. Gabapentin, estrogen, and placebo for treating hot flushes: a randomized controlled trial. Obstet Gynecol 2006;108:41-8.

22. Butt DA, Lock M, Lewis JE, et al. Gabapentin for the treatment of menopausal hot flashes: a randomized controlled trial. Menopause 2008;15:310-18.

23. Pandya KJ, Thummala AR, Griggs JJ, et al. Pilot study using gabapentin for tamoxifen-induced hot flashes in women with breast cancer. Breast Cancer Res Treat 2004;83:87-9.

24. Pandya KJ, Morrow GR, Roscoe JA, et al. Gabapentin for hot flashes in 420 women with breast cancer: a randomised double-blind placebo-controlled trial. Lancet 2005;366:818-24.

25. Loprinzi CL, Kugler JW, Barton DL, et al. Phase III trial of gabapentin alone or in conjunction with an antidepressant in the management of hot flashes in women who have inadequate control with an antidepressant alone: NCCTG N03C5. J Clin Oncol 2007;25:308-12.

26. Elkins G, Marcus J, Stearns V, et al. Pilot evaluation of hypnosis for the treatment of hot flashes in breast cancer survivors. Psychooncology 2007;16:487-92.

27. Elkins G, Marcus J, Stearns V, et al. Randomized trial of a hypnosis intervention for treatment of hot flashes among breast cancer survivors. J Clin Oncol 2008;26:5022-6.

28. Elkins G, Fisher WI, Johnson AK, et al. Clinical hypnosis in the treatment of postmenopausal hot flashes: a randomized controlled trial. Menopause 2013;20:291-7

29. Sloan JA, Loprinzi CL, Novotny PJ, et al. Methodologic lessons learned from hot flash studies. J Clin Oncol 2001;19:4280-90.

30. Carpenter JS. The hot flash related daily interference scale: a tool for assessing the impact of hot flashes on quality of life following breast cancer. J Pain Symptom Manage 2001;22:979-89.

31. Newton KM, Buist DSM, Keenan NL, et al. Use of alternative therapies for menopause symptoms: results of a population-based survey. Obstet Gynecol 2002;100:18-25.

32. Buettner $\mathrm{C}$, Kroenke $\mathrm{CH}$, Phillips RS, et al. Correlates of use of different types of complementary and alternative medicine by breast cancer survivors in the Nurses' Health Study. Breast Cancer Res Treat 2006;100:219-27.

33. Kessel B, Kronenberg F. The role of complementary and alternative medicine in management of menopause symptoms. Endocrinol Metab Clin North Am 2004;33:717-39. 